\title{
Wind Load and Wind Vibration Response of a TV Tower Based on Force Measurement Test in a Wind Tunnel
}

\author{
Daqiao Xia (iD) and Kangfu Peng \\ Zhongguangjian (Beijing) Tower Mast Safety Technology Co., Ltd, Beijing 100045, China \\ Correspondence should be addressed to Daqiao Xia; 4063194@qq.com
}

Received 17 May 2021; Revised 20 October 2021; Accepted 9 November 2021; Published 29 January 2022

Academic Editor: Kun Lin

Copyright (C) 2022 Daqiao Xia and Kangfu Peng. This is an open access article distributed under the Creative Commons Attribution License, which permits unrestricted use, distribution, and reproduction in any medium, provided the original work is properly cited.

\begin{abstract}
Television towers have a complex aerodynamic shape and low damping characteristics. As such, the wind load and the wind induction response are key factors in their design and maintenance. To study these two parameters of high-rise TV towers, a wind tunnel force test was conducted at a height of $280 \mathrm{~m}$ using the Foshan TV tower as a model. The TV tower model was divided into seven detachable sections. Three wind fields with different turbulence intensities were simulated in the wind tunnel, corresponding to different sections, and lateral force tests were performed on each section using a high-frequency dynamic balance. The experimental results were used to calculate the wind-induced response when considering the modal (first three-order) coupled response and were compared with the wind-induced response calculated using the complete quadratic combination (CQC) method. The results revealed that equivalent static wind loads (ESQL) and CQC calculation methods were used in this study, and a higher degree of coincidence was observed, which may be useful in engineering practice.
\end{abstract}

\section{Introduction}

Owing to their aesthetic appeal and extraordinary height, TV towers are generally regarded as landmarks in cities. Many super-tall TV towers have been constructed in recent decades. Prominent examples include the Tokyo Skytree (Japan) with a height of $634 \mathrm{~m}$, the New Guangzhou TV tower (China) with a height of $600 \mathrm{~m}$, the CN Tower (Canada) with a height of $553.3 \mathrm{~m}$, the Ostankion Tower (Russia) with a height of $540 \mathrm{~m}$, and the Milad Telecomm Tower (Iran) with a height of $435 \mathrm{~m}$. With the development of new building materials, an increasing amount of lightweight and high-strength materials are being used in highrise buildings. The complicated aerodynamic shape and low damping render TV towers as wind-sensitive. Therefore, the wind load and the wind-induced response are key considerations in the design and maintenance of TV towers.

Active research is currently being conducted on the wind resistance of high-tower buildings. The original wind resistance design can be traced back to the Eiffel Tower. There are three main ways to study the wind loading of these buildings: wind tunnel tests, computational fluid dynamics analysis, and field measurement. Among them, on-site measurement is the most accurate and direct response, but it is costly. Both wind tunnel tests and CFD can be used to control related parameters for comparative studies. Currently, aeroelastic model vibration tests and rigid model force tests are commonly used in wind tunnels. Reference [1] used a circular cylinder tower rocking model to study the characteristics of crosswind responses. The investigation mainly focused on the vortex vibration of the tower. Reference [2] used a high-frequency force balance and a lightweight tower model to conduct wind tunnel experiments involving Nanjing Tower. The experimental results validated his aerodynamic building block approach. Reference [3] conducted force tests on a lattice tower in Brazil at the Boundary Layer Wind Tunnel Laboratory of the University of Western Ontario (UWO) in Canada and simulated various turbulences in a wind tunnel. The influence of turbulence on wind-induced response was discussed. Reference [4] conducted a wind tunnel test on the new Guangzhou TV Tower. The model was divided into 19 
sections and a force test was conducted. Based on the modal coupling, CQC was applied to random vibration response analysis of the Canton Tower. Reference [5] investigated the wind load and the response of a TV tower considering the wind field environment at the top of a mountain. In particular, equivalent static wind load (ESWL) studies were conducted considering the most unfavorable wind direction angle. Reference [6] provided a hybrid aeroelastic-pressure balance (HAPB) used in wind tunnel and also addressed shortcomings of the classical quasisteady theory in predicting galloping instabilities of slender prisms. Reference [7] used forced vibration to conduct wind tunnel tests on the unstable pressure of the cone model and analyzed the wind pressure distribution and unsteady force coefficients on the model. Reference [8] studied the bipolar behavior, vortex shedding characteristics, and cross-point correlation of the cone structure force systematically. Reference [9] conducted on-site measurements on a steel-framed tower and obtained the dynamic characteristics of the structure. The dynamic response of the tower under wind loading was investigated before and after the attachment of ancillaries. Reference [10] performed long-term health monitoring of a TV tower and processed the results using Palmgren-Miner criteria. Reference [11] described the monitoring of the Stuttgart TV Tower using static and kinematic GPS modes and measured the dynamic characteristics of this structure such as the vibration frequency, damping, and response. Belloi et al. [12] validated the calculated CFD based on wind load measurements during a wind tunnel test. The results also revealed that Eurocodes may underestimate the effect of certain exposures. Reference [13] verified the calculated CFD values using wind load measured using a force test in a wind tunnel for towers with a triangular lattice form. Long-term studies have shown that, due to the complexity of the aerodynamic shape, the wind resistance of high-tower buildings is important and the calculation of wind load and response theory is complex. In this regard, the most common methods are the CQC method used by [4] that considers modal coupling and the SRSS method proposed by [14].

The Foshan TV Tower, with a total height of $238 \mathrm{~m}$, is one of the famous landmarks in Foshan City. Owing to its complexity, narrow structure, and low degree of damping, the tower is highly sensitive to wind loading. It integrates TV signal broadcasting and tourist sightseeing. Therefore, during operation, the structural integrity of the building and the comfort of the visitors are critically important in terms of the wind-induced responses. To study the wind load and response of the Foshan TV Tower, a high-frequency balance technique was used to perform force testing in a wind tunnel. The tower was divided into seven sections, and the force test was performed on each section. In the experiments, wind fields with different turbulence intensities were selected depending on the section of interest. Based on the wind load of the wind tunnel test, the windinduced response of the tower structure was calculated, and the results obtained for different methods (CQC and ESWL) were compared to obtain a reference for similar projects.

\section{Wind Tunnel Test}

2.1. Introduction to the Tower and the Model. The Foshan TV Tower has a height of $236 \mathrm{~m}$, of which the antenna mast on the top of the tower is $71 \mathrm{~m}$ long. The TV tower is a high-rise steel structure, and the main body of the facade is a mesh steel structure, as shown in Figure 1. The bottom layer is connected to the foundation by four limbs, similar to the Eiffel Tower. The lower floor has a circular layout and is used as an office area. The upper region has a spherical section, which is a high-level sightseeing area. Owing to its complex aerodynamic shape and low damping, the Foshan TV Tower is a wind-sensitive building.

Figure 1 shows that the Foshan TV Tower is located in an urban building complex, and the surrounding buildings are much lower than the tower. Moreover, the tower is at a considerable distance from the building complex. This figure also reveals that the top antenna and the region of the tower that connects the two large enclosed sections are very long. A rigid model with a scale of $1: 200$ was fabricated using 3D printing technology for the wind tunnel tests. The model represented the appearance of the TV tower with a high degree of accuracy. The model was divided into seven sections, and the parameters of each section are listed in Table 1.

2.2. Establishment of Turbulence Wind Field. Given that the Foshan TV Tower is close to the center of the city, it should be classified as a C-type terrain according to the "Chinese Building Structure Design Code" [15]. This tower is located in a natural wind field, and the turbulent flow field in the boundary layer changes along the height. It is very tall, and changes in the height along the wind field must be considered. Considering the "Code for Design of Chinese Building Structures" [15] and AIJ [16], the turbulence intensity is given as $I_{u}=0.1 \times\left(z / H_{G}\right)$ $-\alpha_{i}-0.05$, where in the gradient height $H_{G}$ and the exponent of the mean wind speed profile $\alpha_{i}$ are $450 \mathrm{~m}$ and 0.22 according to [15].

To simulate uniform turbulence in the wind tunnel with different intensities, grids with different air permeabilities were used [17], as shown in Figure 2. The experiments were conducted in a closed-circuit type, second test section of the wind tunnel (XNJD-1), with a $2.4 \mathrm{~m}$ (width), $2 \mathrm{~m}$ (height), and $16 \mathrm{~m}$ (length). The turbulence characteristics, including correlation, were measured using the Cobra beat at the model site. For each model section, the height variation range was small. Therefore, the height variation along the wind field was ignored. The Foshan TV Tower is much taller than the surrounding buildings; thus, the influence of the surrounding environment on the wind field can be neglected $[18,19]$. Through constant adjustment of the air permeability of the grille, two grilles and one type of wedge were finally selected to generate turbulence. Three different wind fields were simulated in the laboratory that corresponded to different working conditions, as shown in Table 2. Moreover, not only did the simulated wind field meet the requirements to achieve the appropriate turbulence intensity, but the wind spectrum was also compared 

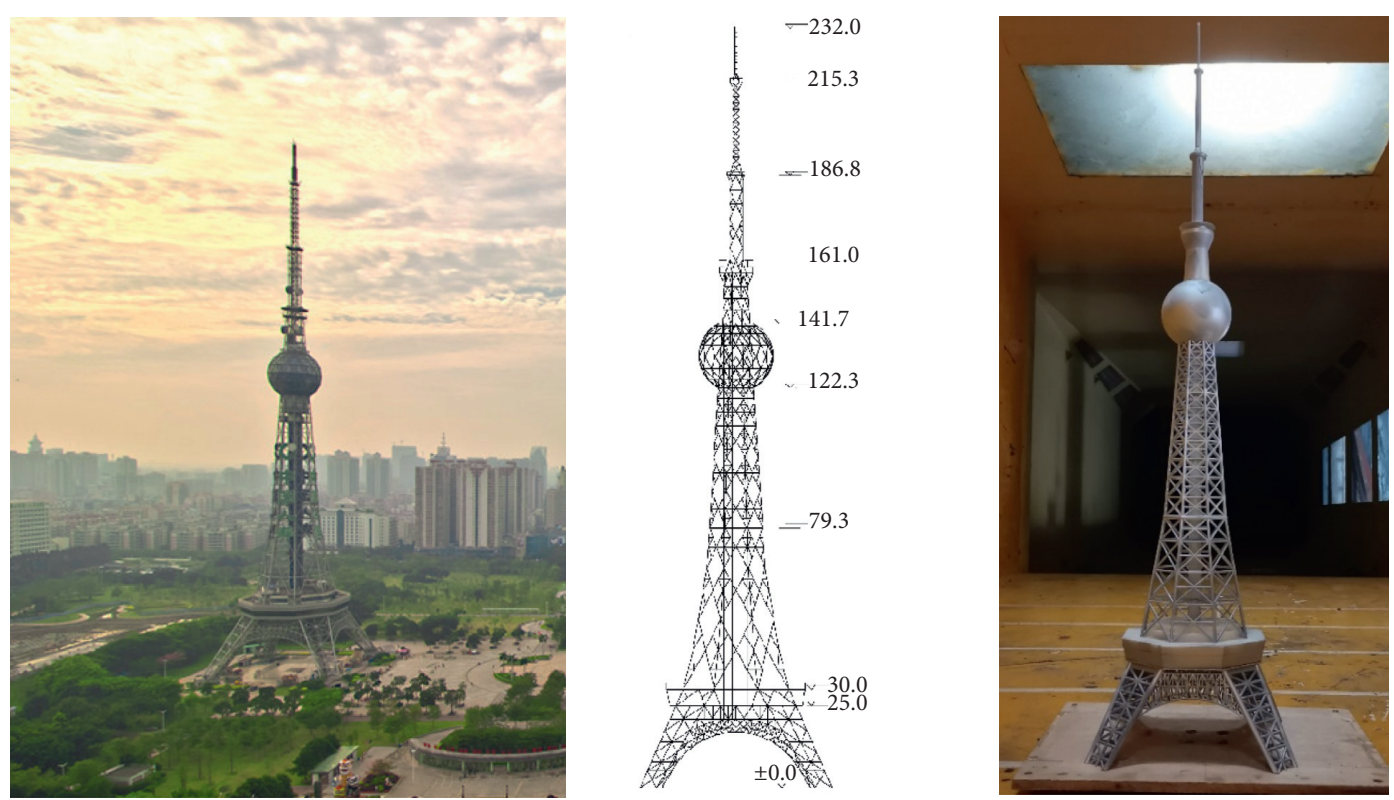

Figure 1: Foshan TV Tower.

Table 1: Parameters of the different sections of the wind tunnel test model.

\begin{tabular}{lccc}
\hline Number & Range of height $(\mathrm{m})$ & Section description & Scale ratio \\
\hline 1 & $0.0-25.0$ & The first nonclosed area of tower body & $1: 200$ \\
2 & $25.0-30.0$ & The first closed area of tower body & $1: 200$ \\
3 & $30.0-79.0$ & The second closed area of tower body & $1: 200$ \\
4 & $79.3-122.3$ & The third closed area of tower body & $1: 200$ \\
6 & $141.7-161.0$ & The fourth closed area of tower body & $1: 200$ \\
7 & $161.0-232.0$ & The antenna part & $1: 200$ \\
\hline
\end{tabular}
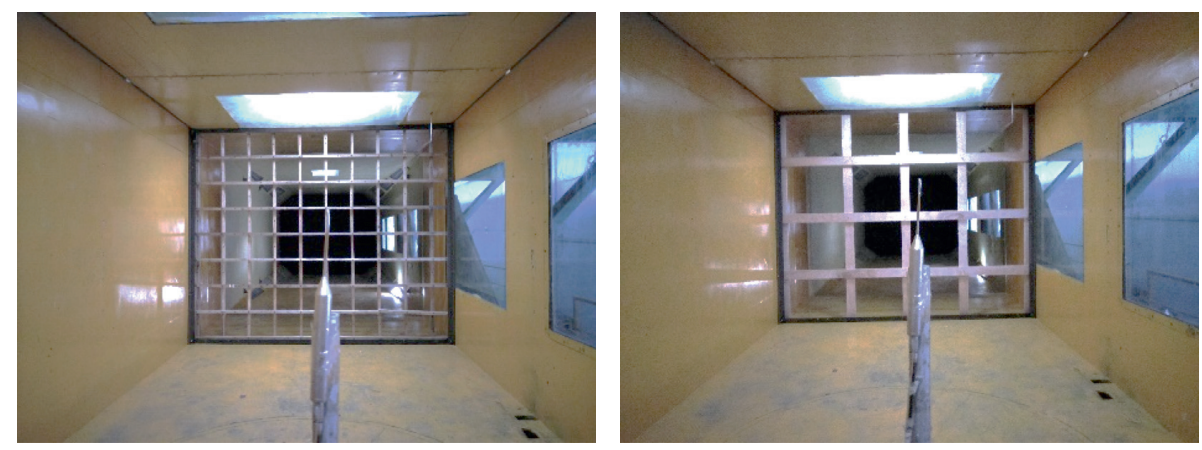

Figure 2: Grids with different air permeability.

TABLE 2: Parameters of the simulated turbulent wind in the tunnel.

\begin{tabular}{lccc}
\hline Height range $(\mathrm{m})$ & Reference height $(\mathrm{m})$ & Number of sectional model & Turbulence intensity (\%) simulated/target \\
\hline $0.0-79.3$ & 40 & $1,2,3$ & $17.7 / 18.0$ \\
$79.3-161.0$ & 120 & $4,5,6$ & $15.2 / 15.0$ \\
$161.0-232.0$ & 195 & 7 & $11.8 / 12.0$ \\
\hline
\end{tabular}

with the recommendations in the specification [20], as shown in Figure 3 (one of them). The results show that the simulated wind field not only meets the required turbulence intensity, but also satisfies the site category specified by the code.
2.3. Force Measurement in the Wind Tunnel. In this study, a rigid model was used for force testing, as shown in Figure 4. The structure of the Foshan TV Tower was narrow. Therefore, if the tower is directly used to test the wind force, a system error is generated. In particular, unlike many TV 


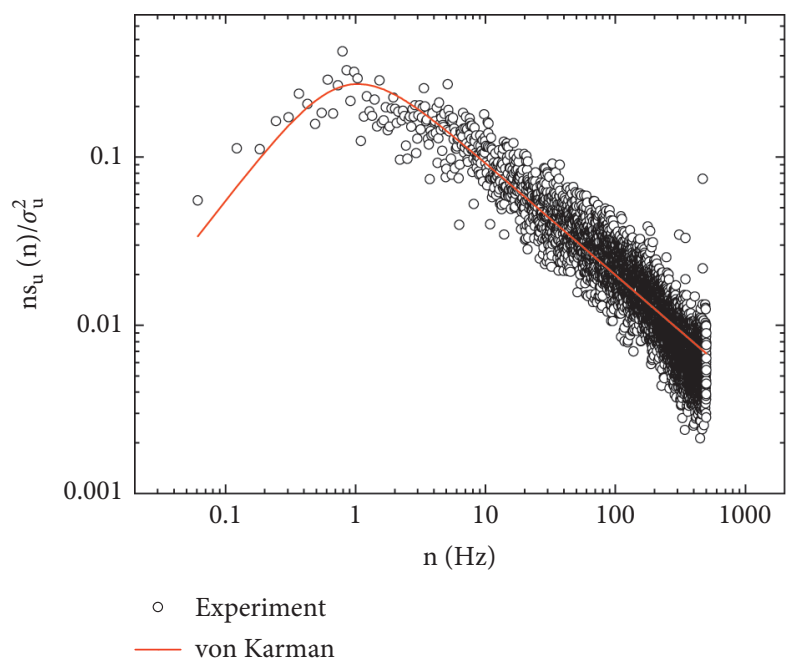

Figure 3: Turbulence wind power spectrum.
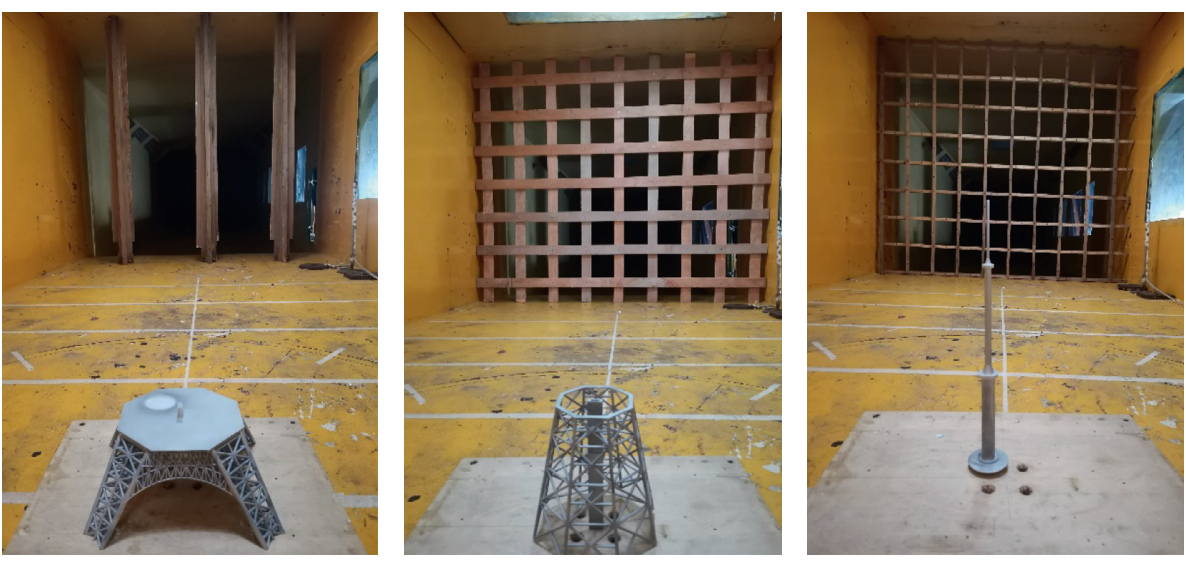

FIGURE 4: Images of the sectional model of the tower in the wind tunnel.

towers, the base of the Foshan Tower has four bearings. In this investigation, the model was designed to be removable and consisted of seven separable sections, with the antenna being a separate section. During the fabrication of models, light and rigid materials should be used to ensure highprecision measurements. The model was $3 \mathrm{D}$ printed, and the material used was rigid, ensuring light weight and high strength. The parameters of the section model used in the wind tunnel test are listed in Table 1.

The force test was conducted in the wind tunnel laboratory of Southwest Jiaotong University (XNJD-1). The positions of the model and the grid are shown in Figure 4. High-frequency dynamic balances (ATI Nano 17) were used for force measurements. Given that the bottom of the Foshan Tower has four supports, a special steel plate was used to connect the high-frequency dynamic balances to the model. The sampling frequency was $1000 \mathrm{~Hz}$ and the sampling time was $60 \mathrm{~s}$, which is dozens of times higher than the natural frequency of the actual structure. The wind tunnel and measuring instruments are shown in Figure 5. The model was installed on a turntable with a diameter of $2.1 \mathrm{~m}$. The direction of the model can be changed to adjust the incident angle of the incoming flow. The wind direction angle range of this test was $-90^{\circ}-90^{\circ}$, as shown in Figure 6. Given that the model was narrow, infrared instruments were used to ensure that the model and the high-frequency dynamic balance were in a vertical state during installation.

In the wind tunnel test, the model size and wind speed are much smaller than the actual situation, the Reynolds number is also smaller than the real condition (Simiu and Scanlan 1996). As to some sections of the models, the effect of Reynolds number can be neglected because of the existence of sharp leading edges. In addition, since the tests were conducted under incoming wind of high turbulence, which will further reduce the effect of Reynolds number on the wind forces, the effect of Reynolds number is considered as negligible for these tests.

2.4. Results of the Wind Tunnel Test. The wind time history results were obtained under different working conditions. To simplify the subsequent calculations, the experimental results were processed without dimensions: 

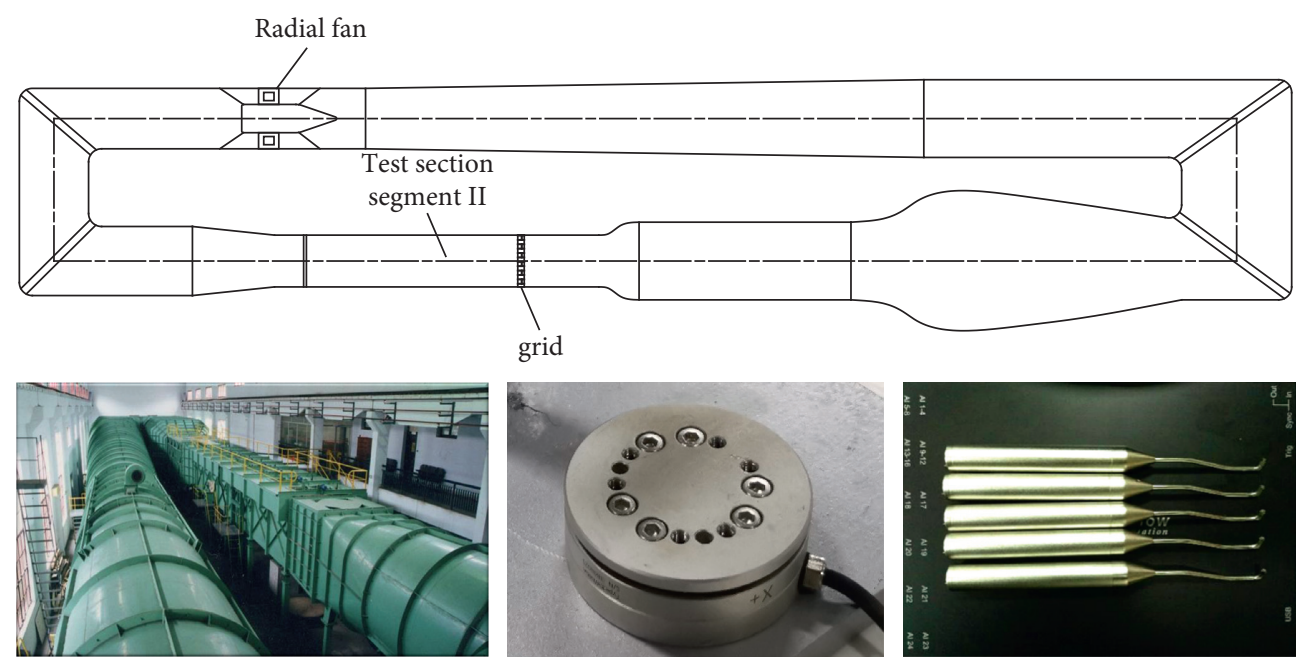

FIGURE 5: Wind tunnel and test equipment.
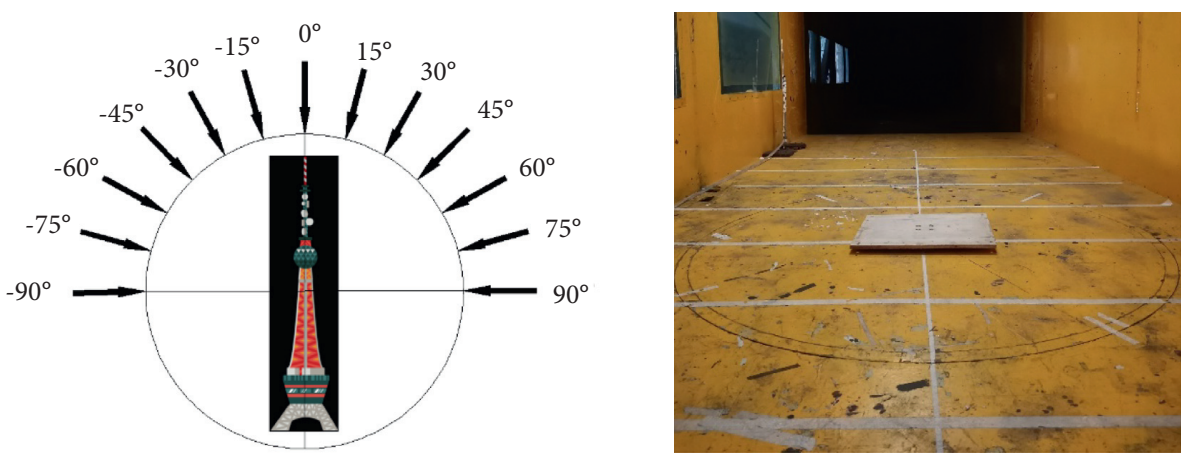

Figure 6: Definitions of the coordinate system and wind direction.

$$
\begin{aligned}
\mu_{F_{X, Y}}\left(t_{p}\right) & =\frac{2 \cdot F_{(X, Y)_{p}}\left(t_{p}\right)}{\rho U_{p}^{2} H_{p} D_{p}}, \\
\mu_{M_{Z}}\left(t_{p}\right) & =\frac{2 \cdot M_{Z_{p}}\left(t_{p}\right)}{\rho U_{p}^{2} H_{p} D_{p}},
\end{aligned}
$$

where $F_{(X)}, F_{(Y)_{p}}$ denote the time histories of the wind forces and the torsional wind force, respectively, and $U, V$, and $W$ denote the longitudinal, lateral, and vertical directions, respectively. The subscript $p$ represents the number of sectional models. $H_{p}$ is the height of the sectional model, and $D_{p}$ is the characteristic length of the sectional model. Given that the TV tower consists of different sections, $D_{p}$ depends on the size at the center of each section.

In this study, a uniform test wind speed was used for all the segment moduli, and the wind speed varied with height in the actual building. So we introduced the reduced time scale $\lambda_{t}\left(\lambda_{t}=\lambda_{L} / \lambda_{V}\right)$ : the ratio between the length scale and the speed scale [4]. In this way, the time histories of the actual aerodynamic force coefficient, $\mu_{F_{X}}(t), \mu_{F_{Y}}(t)$, and $\mu_{M_{Z}}(t)$, can be obtained by reference to $\lambda_{t}$. The peak factor $g$ is introduced here to calculate the maximum and minimum values of the mean and RMS values of the aerodynamic force coefficient.

$$
\begin{aligned}
& \mu_{M_{Z, \text { min }}}=\mu_{M_{Z, \text { mean }}}-g \mu_{M_{Z, \mathrm{rms}}}, \\
& \mu_{F_{X, Y, \text { min }}}=\mu_{F_{X, Y \text {, mean }}}-g \mu_{F_{X, Y, \mathrm{rms}}}, \\
& \mu_{M_{Z, \text { max }}}=\mu_{M_{Z, \text { mean }}}+g \mu_{M_{Z, \mathrm{rms}}}, \\
& \mu_{F_{X, Y, \max }}=\mu_{F_{X, Y \text {, mean }}}+g \mu_{F_{X, Y, \mathrm{rms}}},
\end{aligned}
$$

where $g=3.5$.

The wind force acting on a TV tower can be calculated according to the following aerodynamic force coefficients:

$$
\begin{aligned}
F_{X_{j} Y_{j}}(t) & =\frac{1}{2} \cdot \rho U_{Y_{j}}^{2} H_{j} D \mu_{F_{X} F_{Y}}(t), \\
M_{z_{j}}(t) & =\frac{1}{2} \cdot \rho U_{z_{j}}^{2} H_{j} D \mu_{\mathrm{Mz}}(t), \\
U_{z_{j}} & =U_{G}\left(\frac{Z_{j}}{H_{G}}\right)^{\alpha},
\end{aligned}
$$

where $F_{X_{j} Y_{j}}(t)$ is the time history of the lateral wind force in the $X$ - and $Y$-directions. $F_{X_{j} Y_{i}}(t)$ is the time history of the torsional moment around $Z$. $U_{Y_{j}}$ is the actual wind speed at level $Z_{j} . H_{G}$ and $\alpha$ are the gradient height and exponent of the mean wind speed profile, respectively. $\alpha$ is the actual dimension. 

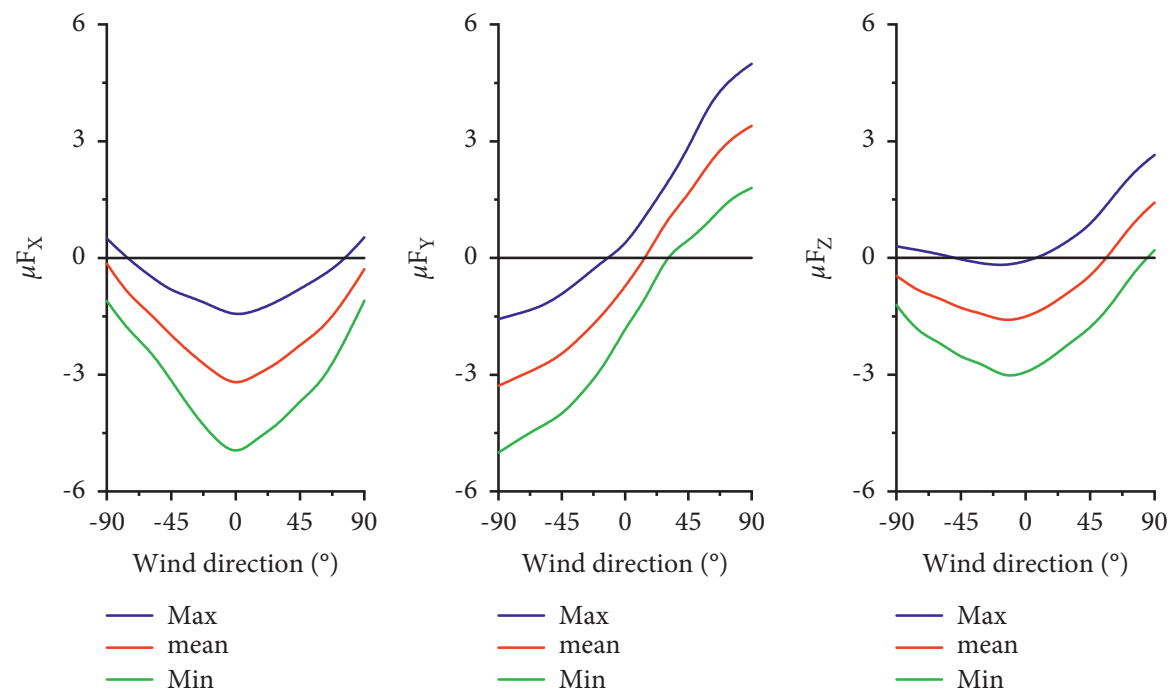

(a)
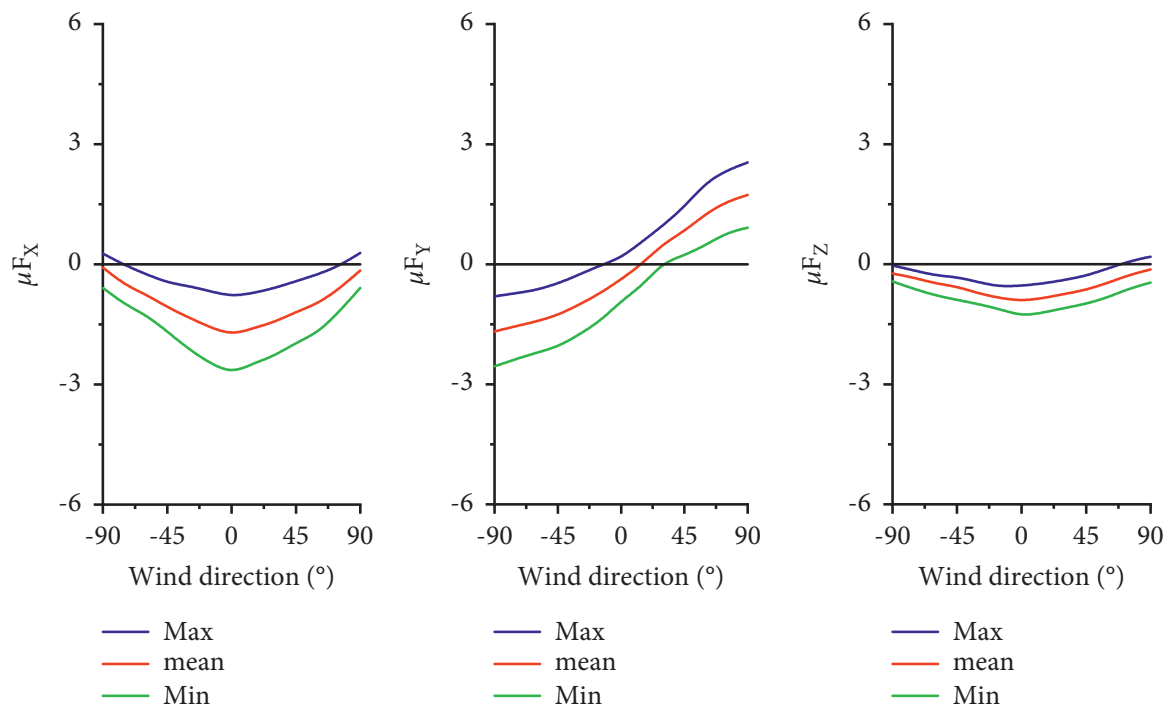

(b)
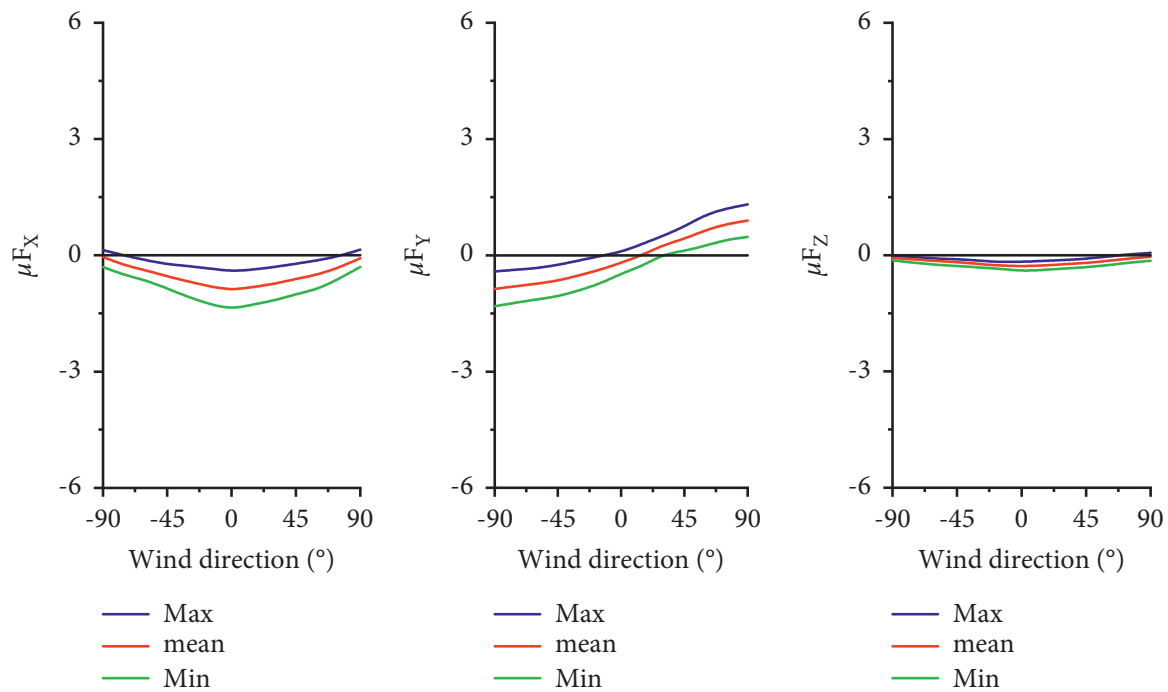

(c)

Figure 7: Variations of mean, maximum, and minimum wind force coefficients with wind direction for the different sections. (a) Section 1, (b) Section 4, and (c) Section 7. 

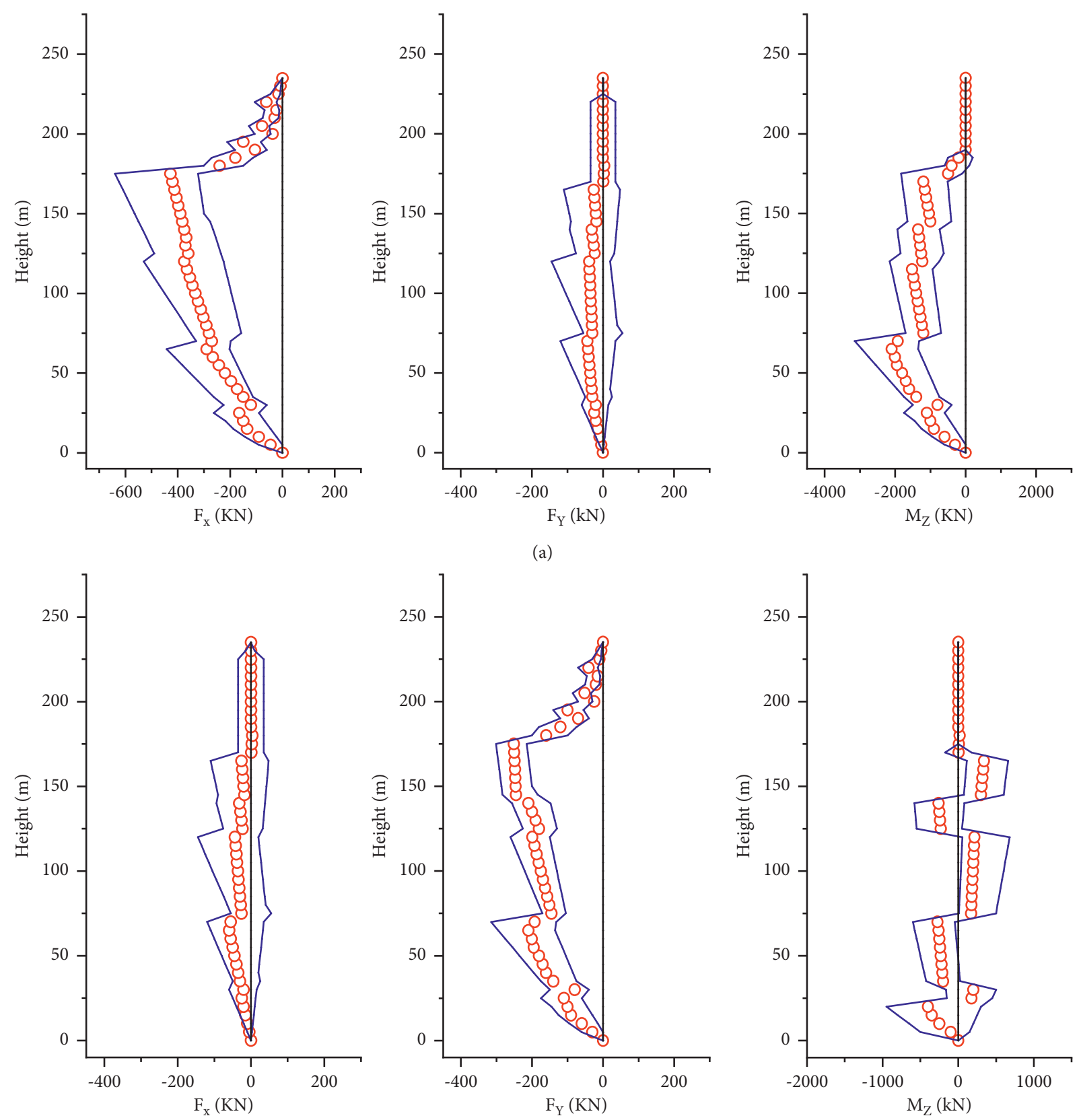

(b)

FIgURe 8: Distributions of mean, maximum, and minimum wind forces along the tower. (a) $0^{\circ}$ wind direction, (b) -90 -degree wind direction.

According to the preceding theory, the relationship between the force coefficient, wind direction, and wind value along the height of the tower can be obtained. The calculation results for typical tower sections are shown in Figures 7 and 8. Figure 7 shows the force coefficient results for the 1st, 4 th, and 7 th sections. It can be observed that the force coefficient changes with the wind direction. When the wind direction foot was $0^{\circ}$, the wind coefficient in the $X$-direction was the largest. When the wind direction angle was $-90^{\circ}$ and $90^{\circ}$, the force coefficient in the $Z$-direction was very large. Moreover, there was a large difference between the force coefficient obtained for the top of the tower and the force coefficient of other towers.

Figure 8 shows the RMS wind force results (for a wind speed of $32.2 \mathrm{~m} / \mathrm{s}$ and a return period of 100 years) for typical wind directions. The figure shows the maximum, minimum, and mean values. The results show that the change of the wind force (RMS) with the wind direction is consistent with the wind coefficient. When the wind direction angle was $0^{\circ}$, the absolute value of the average wind force in the $X$-direction had a maximum value; when the wind direction angle increased to $-90^{\circ}$, the mean wind 


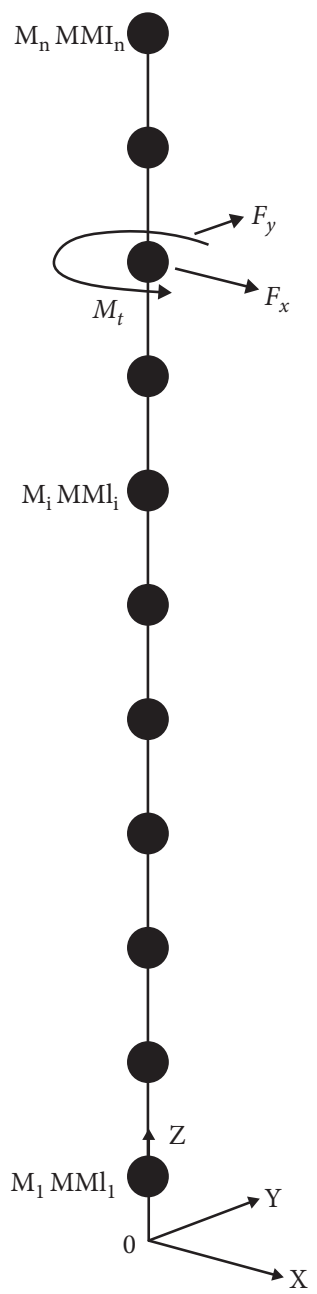

Figure 9: A multiple-degree-of-freedom system with lumped masses in series.

force in the $X$-direction gradually approached 0 . When the wind direction angle was $0^{\circ}$, the absolute value of the mean wind force in the $X$-direction was approximately 0 , and when the wind direction angles were $90^{\circ}$ and $-90^{\circ}$, the mean wind force in the $X$-direction gradually increased. Moreover, the wind force (RMS) of the tower was not continuous, owing to its complex aerodynamic shape, especially the completely closed and the open regions. It can be seen from the results that the results were not completely symmetrical at different wind attack angles. On the one hand, the high-rise structure was inevitably eccentric in the test, and on the other hand, the turbulence is difficult to achieve complete uniformity.

\section{Wind-Induced Responses and Equivalent Static Wind Loads}

Given that the Foshan TV Tower is narrow, it can be regarded as a linear structure when considering the windinduced response. A mechanical model of a multi-degree-offreedom lumped mass system is shown in Figure 9. A finite element model was established to extract the dynamic characteristics of the structure.

3.1. Method for Calculating Equivalent Static Wind Load. The equation of motion can be expressed as follows:

$$
[M]\{\ddot{\delta}\}+[C]\{\dot{\delta}\}+[K]\{\delta\}=[R]\{F(t)\},
$$

where $[M],[C]$, and $[K]$ are the mass matrix, damping matrix, and stiffness matrix, respectively, and $\{\delta\},\{\dot{\delta}\},\{\ddot{\delta}\}$ are the vectors of displacement, velocity, and acceleration, respectively. The random wind load $\{F(t)\}$ can be determined as follows:

$\{F(t)\}=\left(F_{X_{1}}(t), F_{Y_{1}}(t), M_{Z_{1}}(t), \ldots, F_{X_{\mathrm{mf}}}(t), F_{Y_{\mathrm{mf}}}(t) M_{Z_{\mathrm{mf}}}(t)\right)^{T}$.

By evaluating the response power spectral density matrix using the CQC method, we have

$\left[S_{\delta \delta}(w)\right]=[\phi][H]^{*}[\phi]^{T}[R]\left[S_{\mathrm{FF}}(w)\right][R]^{T}[\phi][H][\phi]^{T}$, 

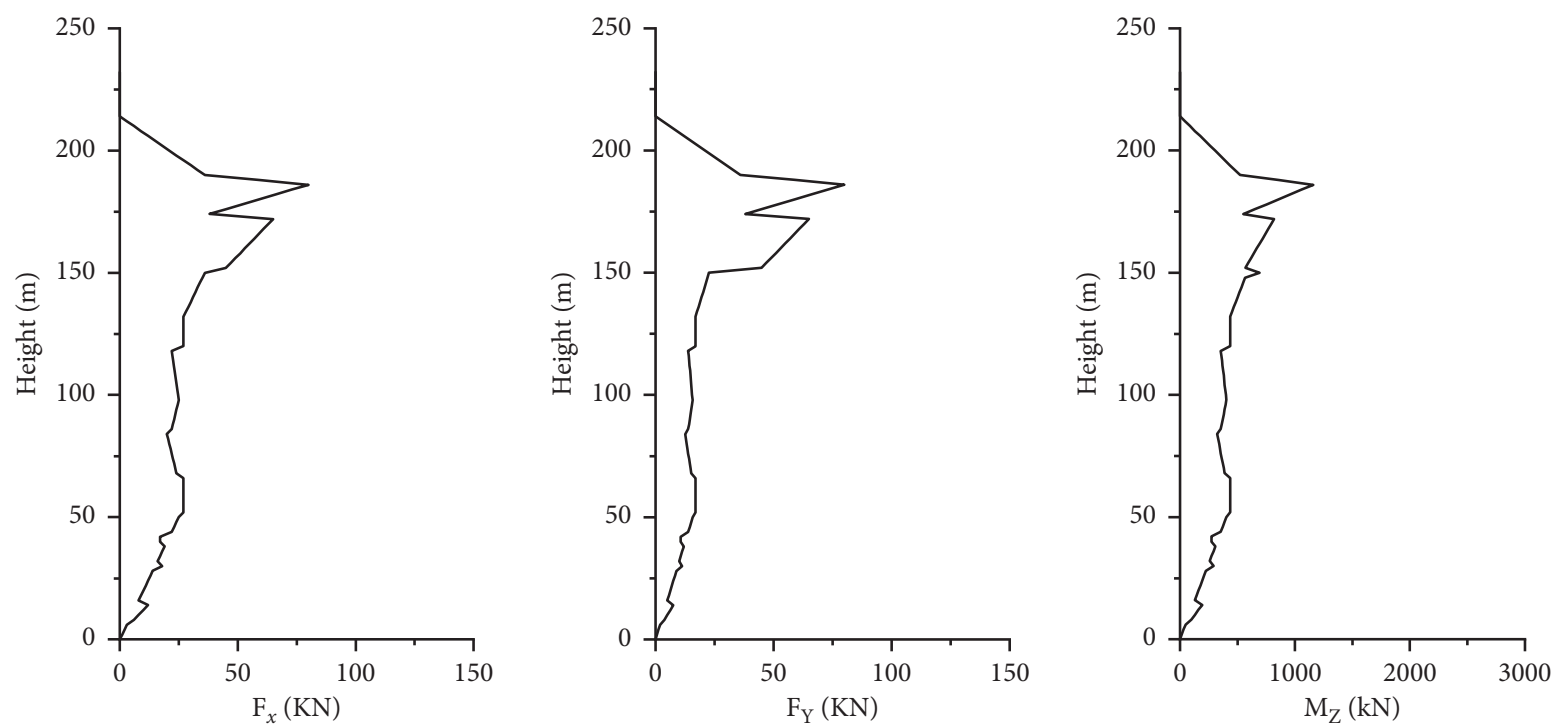

FIgURE 10: Equivalent static wind loads (corresponding to RMS of displacement, $90^{\circ}$ wind direction, 100 years return period).
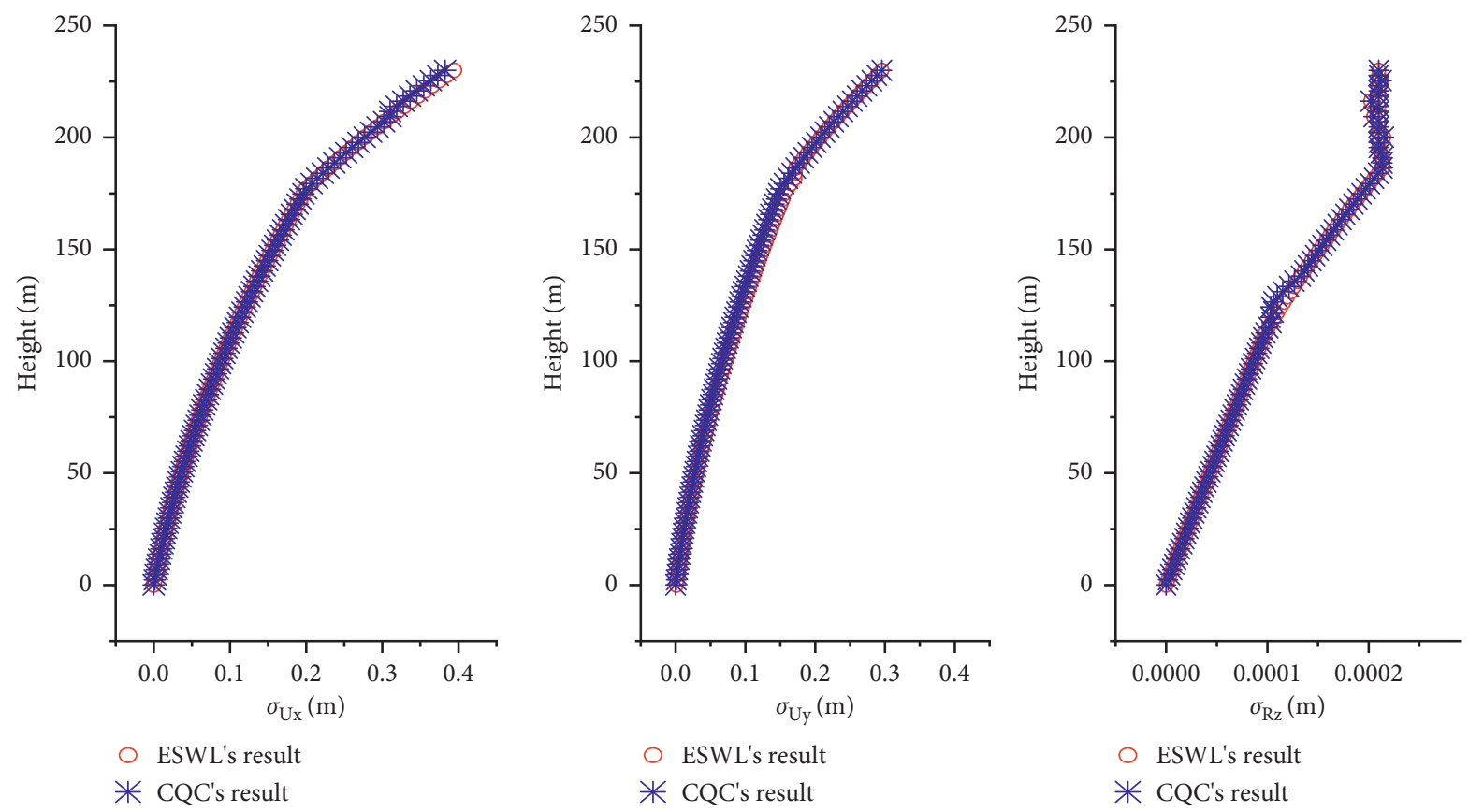

FIGURE 11: RMS displacement of structure ( $90^{\circ}$ wind direction, 100 years return period).

where $[H]$ is the matrix of transfer functions, $[\phi]$ is the matrix of mode shapes, and $[\phi]$ is the force spectrum matrix. The CQC method involves all modal coupling terms that consider the mutual influence effects between the mode shapes. The RMS response of displacement and acceleration can be derived by integrating the power spectrum as follows:

$$
\begin{aligned}
& \sigma_{\delta}(z)=\sqrt{\int_{0}^{\infty} S_{\delta \delta}(w) \mathrm{d} w}, \\
& \sigma_{\tilde{\delta}}(z)=\sqrt{\int_{0}^{\infty} w^{4} S_{\delta \delta}(w) \mathrm{d} w} .
\end{aligned}
$$

For buildings with noncoupled vibration modes, only one term was included in the generalized force, and the other two terms were zero.

3.2. Method for Calculating the Equivalent Static Wind Load. In this study, the structural inertial force method was used to calculate the equivalent static wind load $[4,21]$ as follows:

$$
f_{i}^{e}(z)=k_{i}(z) \sqrt{\sum_{j=1}^{M}\left[m_{i}(z) w_{j}^{2} \sigma_{j} \phi_{\mathrm{ij}}(z)\right]^{2}},
$$




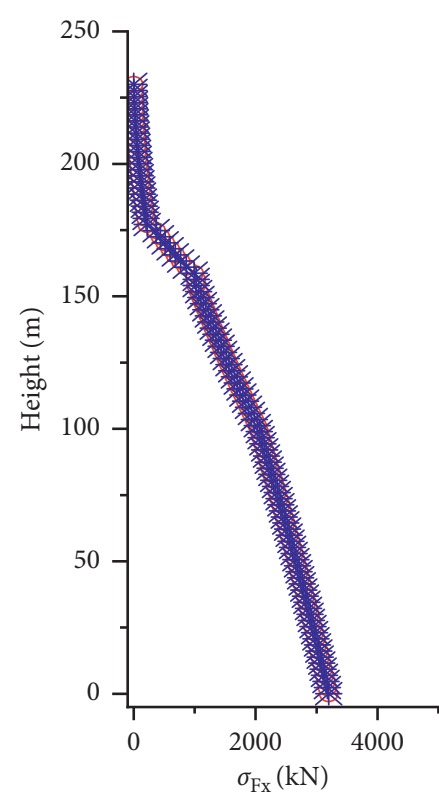

○ ESWL's result * CQC's result

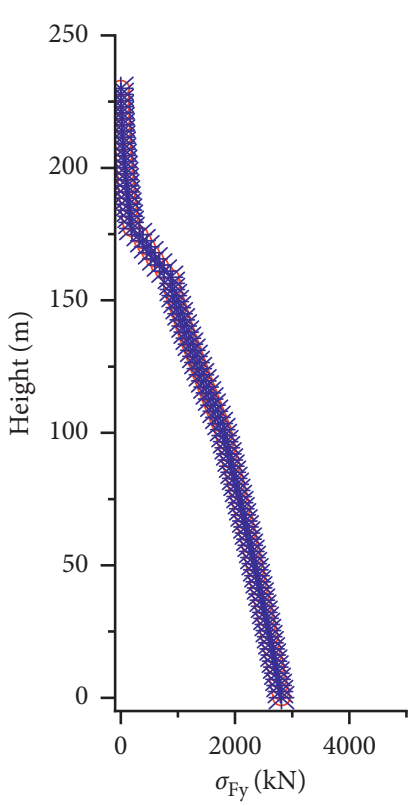

○ ESWL's result * CQC's result

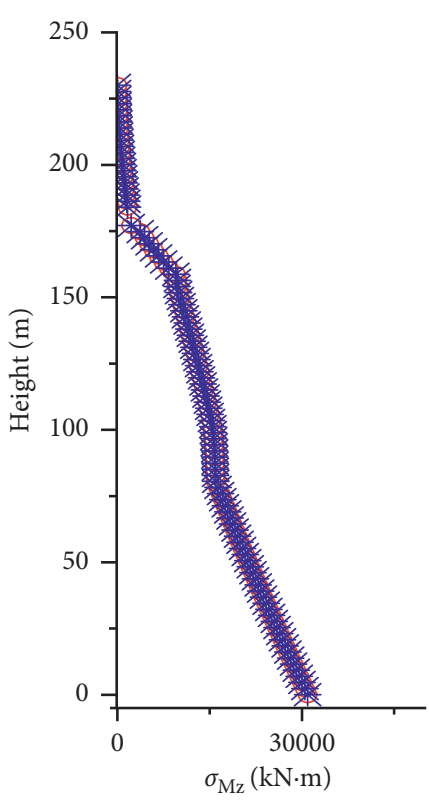

○ ESWL's result * CQC's result
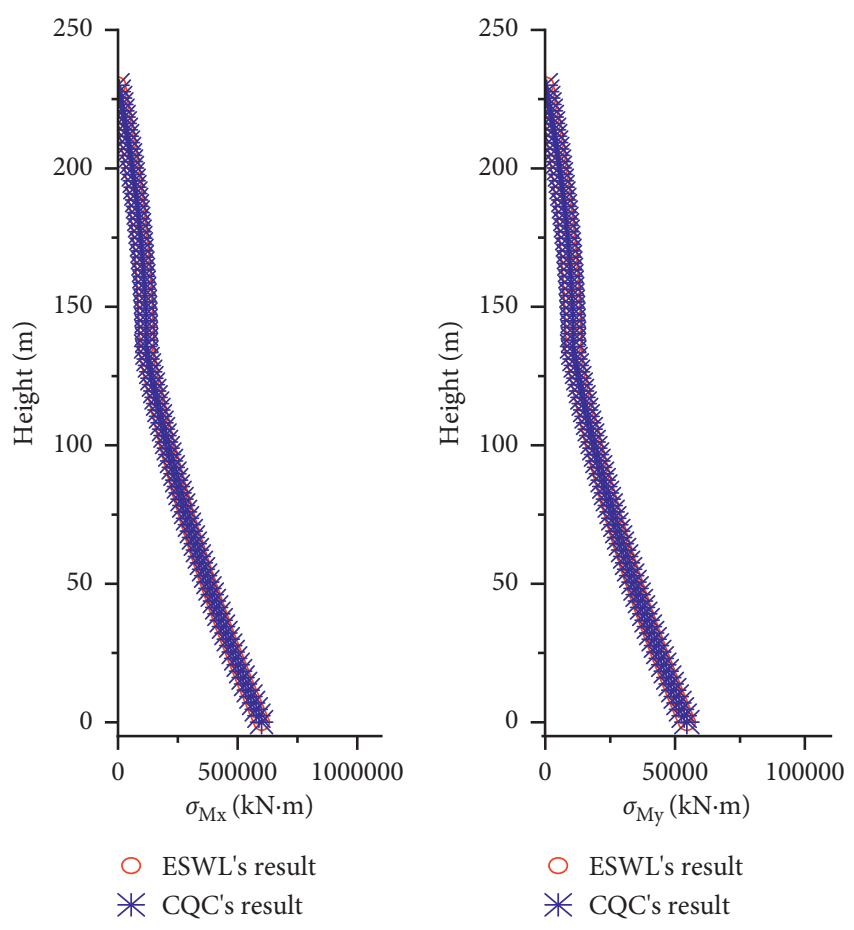

FIGURE 12: RMS internal force of structure $\left(90^{\circ}\right.$ wind direction, 100 years return period). 
where $i=X, Y, R_{Z}, f_{i}^{e}(z)$ is the equivalent static wind load, $\sqrt{\sum_{j=1}^{M}\left[m_{i}(z) w_{j}^{2} \sigma_{j} \phi_{\mathrm{ij}}(z)\right]^{2}}$ is the inertial force of the tower, $k_{i}(z)$ is the adjustment coefficient of the inertial load that varies along the height, $m_{i}(z)$ is the distributed mass or distributed mass moment of inertia, $w_{j}$ is the $j$ th natural frequency, and, $\phi_{\mathrm{ij}}(z)$ is the mode shape. Only the first three modes were considered in this investigation.

\section{Results of Wind-Induced Responses and Equivalent Static Wind Loads}

Based on the theory described in the previous section, we counted the test results. Considering the symmetry of the structure, the range of the wind direction angle was set to $-90^{\circ}-90^{\circ}$ with an interval of $15^{\circ}$.

The equivalent static wind load distributed along the tower was obtained by distributing the static wind load along the TV tower according to the mass distribution ratio, as shown in Figure 10. This figure shows that the equivalent wind load is very small at the bottom and gradually increases with height. In the office area at the bottom and the sightseeing area at the top, the equivalent wind load is relatively large owing to the closed structure. Especially in sightseeing areas, this result should arouse the attention of design and maintenance personnel. In addition, it was observed that the equivalent mass changes in the three directions of $X, Y$, and $Z$ were similar, because the bridge tower was symmetrical. It is also evident that the equivalent static wind load on each section also changes.

The response distributions were calculated when the equivalent static wind loads were exerted on the TV tower as exterior loads, as shown in Figures 11 and 12. The calculated RMS responses using the CQC method are also presented in Figures 11 and 12, which are regarded as the exact values in this study. A comparison of the results obtained using the two methods indicates that the equivalent static wind loads presented in this study have a high degree of precision and can be used in the structural design of the tower.

\section{Concluding Remarks}

As a well-known landmark, the Foshan TV Tower consists of both the office area of the TV station and a high-rise platform for tourists. Owing to its narrow structure, its wind resistance is important, especially in terms of later operations and maintenance. As such, conventional test methods used to measure the wind-induced response and equivalent static wind load will be significantly affected. In this investigation, a novel method that uses segmented force measurements to examine wind-induced responses was used to calculate the equivalent wind load. This method considers the coupling of multiple modes. However, to reduce the workload, only the first three modes of coupling were considered. The results were compared with that of the CQC method, and the main findings are as follows:

(1) To accurately measure and calculate the response and equivalent wind load of the TV tower, the narrow structure was subjected to a wind tunnel test of segmental force measurement. This test method is applicable to narrow structures.

(2) Three different turbulent wind fields were simulated in a wind tunnel test using different grids and wedges. The turbulence degree of the wind field was consistent with the change in the wind field with height.

(3) The force measurement results of the wind tunnel test showed that the wind force in the downwind direction was the largest, whereas the force in the crosswind direction was zero, and the wind direction angle had a periodic influence on the wind force. When the wind angle was $0^{\circ}$, the wind force was the highest in the downwind direction. The force measurement results also confirmed that the wind force in the closed space was greater than that in the open space. This is due to the relatively larger area of the closed space, which is particularly noteworthy.

(4) This study further confirmed that the ESWL method and the CQC method have similar structural responses in turbulence, and both can be used for tower structure design. Considering the convenience, ESWL method is more suitable for engineering calculation.

\section{Data Availability}

The data used to support the findings of this study are available from the corresponding author upon request.

\section{Conflicts of Interest}

The authors declare that they do not have any commercial or associative interest that represents a conflict of interest in connection with the work submitted.

\section{Acknowledgments}

This work was supported by the National Natural Science Foundation of China under the grant number 52068020. The authors are grateful for the test equipment provided by the Laboratory for Wind Engineering of Southwest Jiaotong University.

\section{References}

[1] K. Tetsuya, W. Toshihiro, F. Yozo, and K. Kichiro, "An experimental study on vortex-induced vibration of a circular cylinder tower at a high wind speed," Journal of Wind Engineering and Industrial Aerodynamics, vol. 69-71, 1997.

[2] A. Kareem, S. Kabat, and F. L. Haan, "Aerodynamics of nanjing tower:," Journal of Wind Engineering and Industrial Aerodynamics, vol. 77-78, no. 5, pp. 725-739, 1998.

[3] C. F. Carril, N. Isyumov, and R. M. L. R. F. Brasil, "Experimental study of the wind forces on rectangular latticed communication towers with antennas," Journal of Wind Engineering and Industrial Aerodynamics, vol. 91, no. 8, pp. 1007-1022, 2003. 
[4] X. Zhou, P. Huang, M. Gu, L. Zhu, and H. Pan, "Wind loads and wind-induced responses of guangzhou new tv tower," Advances in Structural Engineering, vol. 13, no. 4, pp. 707-726, 2010.

[5] S. Ke, H. Wang, Y. Ge, L. Zhao, and S. Cao, "Equivalent static wind loads analysis of tall television towers considering terrain factors of hilltops based on force measurement experiment. Structural engineering and mechanics," International Journal, vol. 63, 2017.

[6] Z. Chen, K. T. Tse, K. Kwok, A. Kareem, and B. Kim, "Measurement of unsteady aerodynamic force on a galloping prism in a turbulent flow: a hybrid aeroelastic-pressure balance," Journal of Fluids and Structures, vol. 102, Article ID 103232, 2021.

[7] Z. Chen, H. Huang, Y. Xu, K. T. Tse, and Y. Wang, "Unsteady aerodynamics on a tapered prism under forced excitation," Engineering Structures, vol. 240, Article ID 112387, 2021.

[8] Z. Chen, X. Fu, Y. Xu, C. Y. Li, B. Kim, and K. T. Tse, "A perspective on the aerodynamics and aeroelasticity of tapering: partial reattachment," Journal of Wind Engineering and Industrial Aerodynamics, vol. 212, 2021.

[9] M. J. Glanville and K. C. S. Kwok, "Dynamic characteristics and wind induced response of a steel frame tower," Journal of Wind Engineering and Industrial Aerodynamics, vol. 54-55, no. 2, pp. 133-149, 1995.

[10] M. Pirner and O. Fischer, "Long-time observation of wind and temperature effects on tv towers," Journal of Wind Engineering and Industrial Aerodynamics, vol. 79, 1999.

[11] P. Breuer, T. Chmielewski, P. Górski, E. Konopka, and L. Tarczyński, "The Stuttgart TV Tower - displacement of the top caused by the effects of sun and wind," Engineering Structures, vol. 30, no. 10, pp. 2771-2781, 2008.

[12] M. Belloli, L. Rosa, and A. Zasso, "Wind loads on a high slender tower: numerical and experimental comparison," Engineering Structures, vol. 68, no. jun.1, pp. 24-32, 2014.

[13] F. Yang, H. Dang, H. Niu, H. Zhang, and B. Zhu, "Wind tunnel tests on wind loads acting on an angled steel triangular transmission tower," Journal of Wind Engineering and Industrial Aerodynamics, vol. 156, pp. 93-103, 2016.

[14] S. T. Ke, Y. J. Ge, L. Zhao, and Y. Tamura, "A new methodology for analysis of equivalent static wind loads on superlarge cooling towers," Journal of Wind Engineering and Industrial Aerodynamics, vol. 111, pp. 30-39, 2012.

[15] Gb 50009-2012, Load Code for the Design of Building Structures, China Architecture and Building Press, Beijing, China, 2012.

[16] Architectural Institute of Japan, AIJ Recommendations for Loads on Building, AIJ, Tokyo, 2004.

[17] M. Li, M. Li, and Y. Sun, "Effects of turbulence integral scale on the buffeting response of a long-span suspension bridge," Journal of Sound and Vibration, vol. 490, Article ID 115721, 2021.

[18] Z. N. Xie and M. Gu, "Mean interference effects among tall buildings," Engineering Structures, vol. 26, no. 9, pp. 1173-1183, 2004.

[19] M. Gu, Z. N. Xie, and P. Huang, "Along-wind dynamic interference effects of tall buildings," Advances in Structural Engineering, vol. 8, no. 6, pp. 623-636, 2005.

[20] J. C. Kaimal, J. C. Wyngaard, Y. Izumi, and O. R. Coté, "Spectral characteristics of surface-layer turbulence," Quarterly Journal of the Royal Meteorological Society, vol. 98, 1972.

[21] Z. Yin, G. Ming, and X. Haifan, "Alongwind static equivalent wind loads and responses of tall buildings. part i: unfavorable distributions of static equivalent wind loads," Journal of Wind Engineering and Industrial Aerodynamics, vol. 79, 1999. 